\title{
Bounds on the mean residual lifetime of progressive type II right censored order statistics
}

Mohammad Z Raqab

Correspondence: mraqab@stat.kuniv.edu Department of Statistics and Operations Research, Kuwait University, P.O. Box 5969 Safat, 13060 Kuwait City, Kuwait

\begin{abstract}
In this paper, we present some sharp upper bounds on the deviations of the mean residual lifetime of progressive type $\|$ right censored order statistics from the mean residual lifetime, $m(t)=E\left(X_{t}\right)=E(X-t \mid X>t)$, for arbitrary $t>0$. We also describe the distributional forms for which the bounds are attained. The obtained bounds are numerically evaluated and compared with other classical rough ones.
\end{abstract}

Keywords: Cauchy-Schwarz inequality; Sharp bounds; Characterization; Greatest convex minorant approximation

AMS subject classifications: $62 \mathrm{G} 30 ; 62 \mathrm{E} 15$

\section{Introduction}

The scheme of progressive type II censored sampling is an important scheme in lifetesting experiments. The experimenter can remove units from a life test at various stages during the experiments, possibly resulting in a saving of cost and of time (see Sen 1986).

Suppose $\mathrm{N}$ units are randomly placed on a life test; at the first failure time of one of the units, a number of $R_{1}$ surviving units is randomly withdrawn from the test; at the second failure time, $R_{2}$ surviving units are selected at random and taken out of the experiment, and so on; finally, at the time of the $n^{\text {th }}$ failure, the remaining $R_{n}=$ $N-R_{1}-\ldots-R_{n-1}-n$ objects are removed. This type of schemes is applied in clinical trials. In the context of life-testing, suppose that $X_{1: n}^{\tilde{R}} \leq \ldots \leq X_{n: n}^{\tilde{R}}$ are the lifetimes of the completely observed units to fail, and that $\tilde{R}=\left(R_{1}, \ldots, R_{n}\right)$ represents the number of units withdrawn at these failure times. The model of ordinary order statistics is contained in the above set-up by choosing $\tilde{R}=(0, \ldots, 0)$ (that is $n=N)$ as censoring scheme, where no withdrawals are made. The type II censored order statistics are obtained by setting $R_{1}=\ldots=R_{n-1}=0$ and $R_{n}=N-n$. For more details on progressive censoring, one can refer to Balakrishnan and Aggarwala (2000) and references therein.

Let $X$ be a lifetime variable with cumulative distribution function (cdf) $F$, probability density function (pdf) $f$ and finite first and second moments. Further, let $t=F^{-1}(p)>0$

(c) 2014 Raqab; licensee Springer. This is an Open Access article distributed under the terms of the Creative Commons Attribution License (http://creativecommons.org/licenses/by/4.0), which permits unrestricted use, distribution, and reproduction in any medium, provided the original work is properly credited. 
$(0 \leq p<1)$ be the $100 p$ th percentile of $F$. The mean and variance of the residual lifetime (MRL) r.v. $X_{t}$ are defined as

$$
\begin{array}{r}
m(t)=\mathbb{E}\left(X_{t}\right)=\mathbb{E}(X-t \mid X>t)=\frac{1}{1-p} \int_{p}^{1}\left[F^{-1}(u)-t\right] d u, \\
\sigma^{2}(t)=\mathbb{E}_{F}\left(X_{t}-m(t)\right)^{2}=\frac{1}{1-p} \int_{p}^{1}\left[F^{-1}(u)-t-m(t)\right]^{2} d u .
\end{array}
$$

The mean residual lifetime $m(t)$ is of interest in many fields such as reliability, survival analysis, actuarial studies, etc. It plays an important role in studying the conditional tail measure of the lifetime data. In fact, $m(t)$ can be considered as the conditional tail measure given that an object did not fail in $(0, t)$. In actuarial studies, the insurer might ignore the losses below the deductible $t>0$ since they do not result in insurance payment (insurer might not be aware of the losses below $t$ ). That is, the mean excess loss function or MRL function $m(t)$ does arise naturally. It is well-known that the MRL function $m(t)$ characterizes the distribution function $F$ uniquely (see, for example, Kotz and Shanbhag (1980)). Indeed, when $X$ is non-negative, then for $t>0$ we have

$$
\bar{F}(t)=\frac{m(0)}{m(t)} \exp \left(-\int_{0}^{t} \frac{1}{m(x)} d x\right)
$$

In the spirit of order statistics, Li and Chen (2004) have discussed the aging properties of the residual life length of $m$-out-of- $n$ system with independent (not necessarily identical) components given that $(n-m)$ th failure has occurred at time $t>0$. The aging properties of the parallel system have been studied by Abouammoh and El-Neweihi (1986). Poursaeed and Nematollahi (2008) have studied the mean past and mean residual life functions of the components of parallel system under double monitoring. Recently, Hashemi et al. (2010) have studied some properties of the residual lifetime of progressively type II right censored order statistics (PCOSs). For comprehensive review and applications of the mean residual function, we refer, for example, to Guess and Proschan (1988), Bairamov et al. (2002), Asadi and Bayramoglu (2005) and Asadi and Bayramoglu (2006).

In fact, there are two concepts for the MRL of PCOSs appeared in the literature. The first one represents the average of remaining waiting time after a certain time $t>0$, to the failure of the $s^{t h}$ item from progressively type II censored sample. Precisely,

$$
M_{s, n}^{\tilde{R}}(t)=\mathbb{E}\left(X_{s: n: N}^{\tilde{R}}-t \mid X_{s: n: N}^{\tilde{R}}>t\right) .
$$

Another related concept of the MRL of PCOSs (Hashemi et al. (2010)) can be defined as follows. Under the condition that exactly $r$ items, have failed at or before time $t$, the MRL of the sth failure time, for $0 \leq r<s \leq n \leq N$, is

$$
M_{s, n}^{*^{\tilde{R}}}(t)=\mathbb{E}\left(X_{s: n: N}^{\tilde{R}}-t \mid X_{r: n: N}^{\tilde{R}} \leq t<X_{r+1: n: N}^{\tilde{R}}\right) .
$$

It should be noticed here that these definitions of MRL are different from the ordinary definition of $m(t)$. The bounds for the moments of order statistics appeared frequently in the literature in the last two decades. Arnold (1985) and Rychlik (1993) presented more general sharp bounds for the maximum and arbitrary combination of order statistics, respectively, of possibly dependent samples. Raqab (2003) developed p-norm bounds for the moments of PCOSs, measured in scale units generated by absolute moments of the parent distribution of a single observation. Recently, Raqab and Rychlik (2011) developed sharp bounds for the MRL of a $m$-out-of- $n$ system using the greatest convex minorant 
approximation combined with the Cauchy-Schwarz inequality. Balakrishnan et al. (2001) applied several methods to derive different bounds for means and variances of PCOSs.

In this paper, we establish sharp bounds for the deviations of the MRL $M_{s, n}^{\tilde{R}}(t)$ of PCOSs from the MRL $m(t)$ of $X$ for fixed $t>0$. The so obtained bounds here are derived based on $L^{2}$ - projection approach combined with the Cauchy-Schwarz inequality. These bounds can be considered as optimal estimates of the residual lifetime of PCOSs. Our work is quite general in nature as the bounds expressed in terms of $m(t)$ and $\sigma(t)$ units and the bounds are valid for all parent distribution functions $F$ such that $F^{-1}(t)=p, 0<p<1$. Evaluations of the resulting bounds for various choices schemes are also presented and compared with other classical ones.

\section{Bounds for MRL of PCOSs}

Let $X_{1}, \cdots, X_{n}$ be the iid random variables (r.v.'s) with $\operatorname{cdf} F, \operatorname{pdf} f$, and finite first and second moments. Assume that $t=F^{-1}(p)(0 \leq p<1)$ is the 100pth percentile of $F$. Now, consider the deviation of the MRL of PCOSs from the MRL function of the parent r.v. $X_{1}$ as

$$
\begin{aligned}
M_{s, n}^{\tilde{R}}(t)-m(t) & =\mathbb{E}\left(X_{s: n: N}^{\tilde{R}}-t \mid X_{s: n: N}^{\tilde{R}}>t\right)-\mathbb{E}_{F}\left(X_{1}-t \mid X_{1}>t\right) \\
& =\int_{p}^{1}\left[F^{-1}(u)-t\right] \frac{f_{s: n: N}^{\tilde{R}}(u)}{1-F_{s: n: N}^{\tilde{R}}(p)} d u-\int_{p}^{1}\left[F^{-1}(u)-t\right] \frac{d u}{1-p} \\
& =\int_{p}^{1}\left[F^{-1}(u)-t-m(t)\right]\left[\frac{f_{s: n: N}^{\tilde{R}}(u)}{1-F_{s: n: N}^{\tilde{R}}(p)}-\frac{1}{1-p}\right] d u,
\end{aligned}
$$

where $f_{r: n: N}^{\tilde{R}}$ and $F_{r: n: N}^{\tilde{R}}$ are the pdf and cdf of the $r t h$ PCOS from standard uniform distribution over unit interval $[0,1]$ of the forms:

$$
\begin{aligned}
& f_{r: n: N}^{\tilde{R}}(u)=C_{r-1} \sum_{i=1}^{r} a_{i, r}(1-u)^{\gamma_{i}-1}, 1 \leq r \leq n, \\
& F_{r: n: N}^{\tilde{R}}(u)=1-C_{r-1} \sum_{i=1}^{r} \frac{a_{i, r}}{\gamma_{i}}(1-u)^{\gamma_{i}}, 1 \leq r \leq n,
\end{aligned}
$$

with

$$
C_{r-1}=\prod_{i=1}^{r} \gamma_{i} ; \gamma_{i}=N-\sum_{j=1}^{i-1} R_{j}-i+1 ; \gamma_{1}=N, \text { and } a_{i, r}=\prod_{\substack{j=1 \\ j \neq i}}^{r}\left(\gamma_{j}-\gamma_{i}\right)^{-1},
$$

(cf., e.g., Kamps and Cramer 2001). The following lemma describe the variability of functions $f_{s: n: N}^{\tilde{R}}(u)$ in $[0,1]$.

Lemma 1 (Balakrishnan et al. (2001)). For $n \geq 2, f_{1: n: N}^{\tilde{R}}(u)$ is decreasing with $f_{1: n: N}^{\tilde{R}}(0)=\gamma_{1}$ and $\lim _{u \nearrow 1} f_{1: n: N}^{\tilde{R}}(u)=0$. For $\gamma_{n}=1, f_{n: n: N}^{\tilde{R}}(u)$ is increasing on $(0,1)$ with $f_{n: n: N}^{\tilde{R}}(0)=0$ and $\lim _{u \nearrow 1} f_{n: n: N}^{\tilde{R}}(u)=\prod_{j=1}^{n-1} \frac{\gamma_{j}}{\gamma_{j}-1}$. If either $(1<s<n)$ or $\left(s=n \geq 2, \gamma_{n}>1\right), f_{s: n: N}^{\tilde{R}}(u)$ is first increasing starting from 0 at 0 , then ultimately decreasing to 0 at 1 . Moreover, each $f_{s: n: N}^{\tilde{R}}(u)$ has a unique maximum in $(0,1)$ at $\varepsilon_{s, n, N}>0$ satisfying

$$
\sum_{i=1}^{s} a_{i, s}\left(\gamma_{i}-1\right)\left(1-\varepsilon_{s, n, N}\right)^{\gamma_{i}-2}=0 .
$$


The following lemma describes the Moriguti's inequality obtained by projecting the integrable function $h$ onto the convex cone of non-decreasing functions in $L^{2}([0,1], d u)$.

Lemma 2 (Projection of $\left.f_{s: n: N}^{\tilde{R}}(u)\right)$. For either $(1<s<n)$ or $\left(s=n \geq 2, \gamma_{n}>1\right)$, there exists a unique $u_{s, n, N} \in(p, 1)$ defined as the solution to equation

$$
\sum_{i=1}^{s} a_{i, s}\left(\frac{\gamma_{i}-1}{\gamma_{i}}\right)(1-u)^{\gamma_{i}}=0, \quad p<u<1,
$$

such that for

$$
\widehat{f_{s: n: N}^{\tilde{R}}}(u)= \begin{cases}f_{s: n: N}^{\tilde{R}}\left(\min \left\{u, u_{s, n, N}\right\}\right), & \text { if } 0<p<u_{s, n, N} \\ \frac{1-F_{s: n: N}(p)}{1-p} & \text { if } u_{s, n, N} \leq p<1\end{cases}
$$

and every non-decreasing function $w \in L^{1}([p, 1], d u)$, we have

$$
\int_{p}^{1} w(u) f_{s: n: N}^{\tilde{R}}(u) d u \leq \int_{p}^{1} w(u) \widehat{f}_{s: n: N}^{\tilde{R}}(u) d u,
$$

with the equality iff

$$
w(u)=\text { const }, \quad u_{s, n, N}<u<1 .
$$

Proof. The result of the above lemma is specifically based on a combination of the statement of Lemma 1 with Moriguti (1953), (Lemma 1). It is enough to show that $\widehat{f}_{s: n: N}^{\tilde{R}}(u)$ is the derivative of the greatest convex minorant (GCM) $\widehat{F}_{s: n: N}^{\tilde{R}}(u)$ of the antiderivative $F_{s: n: N}^{\tilde{R}}(u)$ of $f_{s: n: N}^{\tilde{R}}(u)$. By Lemma 1 , the function $F_{s: n: N}^{\tilde{R}}$ is convex increasing on $\left[0, \varepsilon_{r, n, N}\right)$, and concave increasing on $\left(\varepsilon_{r, n, N}, 1\right]$. Therefore, the GCM of the antiderivative $F_{s: n: N}^{\tilde{R}}(u)-$ $F_{s: n: N}^{\tilde{R}}(p)$ of $f_{s: n: N}^{\tilde{R}}(u), p<u<1$, is defined as the supremum of all convex functions dominated by $F_{s: n: N}^{\tilde{R}}(u)-F_{s: n: N}^{\tilde{R}}(p), p<u<1$ and its form is defined as follows:

$$
\widehat{F}_{s: n: N}^{\tilde{R}}(u)= \begin{cases}F_{s: n: N}^{\tilde{R}}(u)-F_{s: n: N}^{\tilde{R}}(p), & \text { if } p<u \leq u_{s, n, N}, \\ F_{s: n: N}^{\tilde{R}}\left(u_{s, n, N}\right)-F_{s: n: N}^{\tilde{R}}(p) & \text { if } u_{s, n, N}<u<1, \\ +f_{s: n: N}^{\tilde{R}}\left(u_{s, n, N}\right)\left(u-u_{s, n, N}\right), & \end{cases}
$$

for a unique $u=u_{s, n, N} \in\left(p, \varepsilon_{s, n, N}\right)>0$ satisfying

$$
1-F_{s: n: N}^{\tilde{R}}(u)=f_{s: n: N}^{\tilde{R}}(u)(1-u) .
$$

or equivalently Eq. (5). If $p \geq u_{s, n, N}$, then

$$
\widehat{F}_{s: n: N}^{\tilde{R}}(u)=\frac{1-F_{s: n: N}^{\tilde{R}}(p)}{1-p}(u-p), \quad p<u<1 .
$$

Differentiating the function $\widehat{F}_{s: n: N}^{\tilde{R}}$, we complete the proof. Function $\widehat{f}_{s: n: N}^{\tilde{R}}(u)$ is called the projection of $f_{s: n: N}^{\tilde{R}}(u)$ onto the convex cone of nondecreasing functions in $L^{2}([0,1], d u)$.

In fact, the deviation $M_{s, n, N}^{\tilde{R}}(t)-m(t)$ in (3) can be evaluated using the inequalities of the integral of the product of two proportional functions. The Moriguti projection method (see Moriguti (1953)) can be used effectively in developing improved bounds 
of the statistical functional over general families of distributions. Applying the CauchySchwarz inequality to (3), we have for either $(1<s<n)$ or $\left(s=n \geq 2, \gamma_{n}>1\right)$,

$$
\begin{aligned}
M_{s, n, N}^{\tilde{R}}(t)-m(t) \leq & \left\{\int_{p}^{1}\left[F^{-1}(u)-t-m(t)\right]^{2} \frac{d u}{1-p}\right\}^{1 / 2} \\
& \times\left\{(1-p) \int_{p}^{1}\left[\frac{f_{s: n: N}^{\tilde{R}}(u)}{1-F_{s: n: N}^{\tilde{R}}(p)}-\frac{1}{1-p}\right]^{2} d u\right\}^{1 / 2} \\
= & \sigma(t) B_{0}^{\tilde{R}}(s, n, N ; p) .
\end{aligned}
$$

Generally, the right hand side of (8) is not monotonic and then the bound $B_{0}^{\tilde{R}}(s, n, N ; p)$ is not attainable. By (3), (6), and the Cauchy-Schwarz inequality, we get

$$
\begin{aligned}
M_{s, n, N}^{\tilde{R}}(t)-m(t) & \leq \int_{p}^{1}\left[F^{-1}(u)-t-m(t)\right]\left[\frac{\widehat{f}_{s: n: N}^{\tilde{R}}(u)}{1-F_{s: n: N}^{\tilde{R}}(p)}-\frac{1}{1-p}\right] d u, \\
& \leq B_{1}^{\tilde{R}}(s, n, N ; p) \sigma(t),
\end{aligned}
$$

with

$$
B_{1}^{\tilde{R}}(s, n, N ; p)=\left\{(1-p) \int_{p}^{u_{s, n, N}} \frac{\left[f_{s: n: N}^{\tilde{R}}(u)\right]^{2}}{\left[1-F_{s: n: N}^{\tilde{R}}(p)\right]^{2}}+\frac{(1-p)\left(1-u_{s, n, N}\right)\left[f_{s: n: N}^{\tilde{R}}(u)\right]^{2}}{\left[1-F_{s: n: N}^{\tilde{R}}(p)\right]^{2}}-1\right\}^{1 / 2} .
$$

The latter inequality in Eq. (9) becomes equality iff

$F^{-1}(u)-t-m(t)=\alpha\left(\frac{\widehat{f}_{s: n: N}^{\tilde{R}}(u)}{1-F_{s: n: N}^{\tilde{R}}(p)}-\frac{1}{1-p}\right), p \leq u<1$, for some $\alpha>0$.

Note that for $p<u_{s, n, N}$, the right-hand side is nonnegative and nondecreasing as desired. From (7), the former inequality of (9) becomes equality iff the right-hand side of Eq. (11) is a constant on $\left(u_{s, n, N}, 1\right)$. The moment condition

$$
\int_{p}^{1}\left[F^{-1}(u)-t-m(t)\right]^{2} \frac{d u}{1-p}=\sigma^{2}(t)
$$

forces $\alpha=(1-p) \sigma(t) / B_{1}^{\tilde{R}}(s, n, N ; p)$. Plugging it in Eq. (11) and using the fact that $F^{-1}(u) \leq t, 0<u<p$, we readily form the cdf that attains the bound in (10). The nonnegativity jump at $t$ leads to the additional condition given below in (13). Thus we have proven the following theorem.

Theorem 1. For a parent distribution $F$ with finite mean $\mu$, variance $\sigma^{2}$ and either $(1<s<n)$ or $\left(s=n \geq 2, \gamma_{n}>1\right)$ with $p<u_{s, n, N}$, we have

$$
\frac{M_{s, n, N}^{\tilde{R}}(t)-m(t)}{\sigma(t)} \leq B_{1}^{\tilde{R}}(s, n, N ; p)
$$

where $B_{1}^{\tilde{R}}(s, n, N ; p)$ is defined in Eq. (10) with unique $u_{s, n, N} \in\left(p, \varepsilon_{s, n, N}\right)$ solving Eq. (5). Under the additional condition

$$
m(t) \geq\left(1-\frac{(1-p) f_{s: n: N}^{\tilde{R}}(p)}{1-F_{s: n: N}^{\tilde{R}}(p)}\right) \frac{\sigma(t)}{B_{1}^{\tilde{R}}(s, n, N ; p)},
$$


the equality in (12) is attained in limit by the distribution function

$$
F(x)= \begin{cases}F_{0}(x), & \text { if } x<t, \\ p, & \text { if } t \leq x<b_{1}, \\ f_{s: n: N}^{\tilde{R}^{-1}}\left(\frac{1-\tilde{s}_{s: n: N}^{\tilde{R}}(p)}{1-p}\left[B_{1}^{\tilde{R}}(s, n, N ; p)\left(\frac{x-t-m(t)}{\sigma(t)}\right)+1\right]\right), & \text { if } b_{1} \leq x<b_{2}, \\ 1, & \text { if } x \geq b_{2},\end{cases}
$$

where $F_{0}$ is an arbitrary continuous function with $F_{0}(t)=p$, and

$$
\begin{aligned}
& b_{1}=t+m(t)-\left(1-\frac{(1-p) f_{s: n: N}^{\tilde{R}}(p)}{1-F_{s: n: N}^{\tilde{R}}(p)}\right) \frac{\sigma(t)}{B_{1}^{\tilde{R}}(s, n, N ; p)}, \\
& b_{2}=t+m(t)+\left(\frac{(1-p) f_{s: n: N}^{\tilde{R}}\left(u_{s, N, p}\right)}{1-F_{s: n: N}^{\tilde{R}}(p)}-1\right) \frac{\sigma(t)}{B_{1}^{\tilde{R}}(s, n, N ; p)} .
\end{aligned}
$$

Remark 1. Distribution function (14) is a location-scale family of distributions consisting any arbitrary continuous function supported on the left of $t$ with probability $p$, constant supported on $\left(t, b_{1}\right)$, has a smooth component on finite support $\left[b_{1}, b_{2}\right]$, and one atom of measure $\left(1-u_{s, n, N}\right)$ at the right end of the support interval. By the monotonicity of $f_{s: n: N}^{\tilde{R}}(u)$ on $\left(p, u_{s, n, N}\right)$, then the right-hand side of (13) is positive. The arbitrary distribution function $F_{0}$ with $F_{0}(t)=p$ defined on the left tail can be assumed in all other distributions attaining the bounds for the MRL function of PCOSs. In a consequence of that, the distribution function $F$ is not absolutely continuous function. However, it can be approximated by sequences of absolutely continuous functions attaining the bound asymptotically.

Let us consider the extreme progressive type II censored order statistics (i) ( $s=n$, $\left.\gamma_{n}=1\right)$ (ii) $s=1$ (iii) $1<s<n$ or $\left(s=n \geq 2, \gamma_{n}>1\right)$ with $p \geq u_{s, n, N}$. In the former case, it is easily checked that $\left[F_{n: n: N}^{\tilde{R}}(u)\right]^{\prime \prime}=(1-u)^{-1} f_{n-1: n: N}^{\tilde{R}}(u) \geq 0$, and then the antiderivative $F_{s: n: N}^{\tilde{R}}(u)$ of $f_{s: n: N}^{\tilde{R}}(u)$ is convex on $[0,1]$. Therefore, the projection of $f_{s: n: N}^{\tilde{R}}(u)$ is the function itself. Arguments similar to those in the above lines provide the corresponding bound and the distribution function for which the bound is attained. For the second case, the pdf is decreasing and its anti-derivative is concave for $(p, 1)$. Therefore, the GCM for the latter two cases amounts to the linear function

$$
\widehat{F}_{1: n: N}^{\tilde{R}}(u)=(1-p)^{N-1}(u-p), p<u<1,
$$

and then the projection of $f_{s: n: N}^{\tilde{R}}(u)$ is just constant. This turns out that the right-hand side of the $2^{\text {nd }}$ inequality amounts to 0 . In this case, Moriguti's method does not lead to an improved bound and the equality becomes equality iff $F^{-1}(u)-t-m(t)$ is constant on $(p, 1)$. Combining this with the moment condition and the fact that $F(t)=p$, we immediately obtain the distribution function attaining the bound. The so obtained bounds for the extreme cases and their respective marginal distributions are summarized in two subsequent theorems. 
Theorem 2. For $\gamma_{n}=1$, the bound on $\left(M_{n, n, N}^{\tilde{R}}(t)-m(t)\right) / \sigma(t)$ is

$$
B_{1}^{\tilde{R}}(n, n, N ; p)=\left\{(1-p) \int_{p}^{1} \frac{\left[f_{n: n: N}^{\tilde{R}}(u)\right]^{2}}{\left[1-F_{n: n: N}^{\tilde{R}}(p)\right]^{2}}-1\right\}^{1 / 2} .
$$

Under Condition (13) with ( $\left.s=n, \gamma_{n}=1\right)$, the bound is attained in the limit by continuous distribution functions converging to the following cdf

$$
F(x)= \begin{cases}F_{0}(x), & \text { if } x<t, \\ p, & \text { if } t \leq x<c_{1}, \\ f_{n: n: N}^{\tilde{R}^{-1}}\left(\frac{1-F_{n: n: N}^{\tilde{R}}(p)}{1-p}\left[B_{1}^{\tilde{R}}(n, n, N ; p)\left(\frac{x-t-m(t)}{\sigma(t)}\right)+1\right]\right), & \text { if } c_{1} \leq x<c_{2}, \\ 1, & \text { if } x \geq c_{2},\end{cases}
$$

where $F_{0}$ is an arbitrary continuous function with $F_{0}(t)=p$, and

$$
\begin{aligned}
& c_{1}=t+m(t)-\left(1-\frac{(1-p) f_{n: n: N}^{\tilde{R}}(p)}{1-F_{n: n: N}^{\tilde{R}}(p)}\right) \frac{\sigma(t)}{B_{1}^{\tilde{R}}(n, n, N ; p)}, \\
& c_{2}=t+m(t)+\left(\frac{(1-p) \prod_{j=1}^{n-1} \frac{\gamma_{j}}{\gamma_{j}-1}}{1-F_{n: n: N}^{\tilde{R}}(p)}-1\right) \frac{\sigma(t)}{B_{1}^{\tilde{R}}(n, n, N ; p)} .
\end{aligned}
$$

Theorem 3. If either $s=1$ or $(1<s<n)$ with $\left.p \geq u_{s, n, N}\right)$ or $\left(s=n \geq 2, \gamma_{n}>1\right)$ with $p \geq u_{s, n, N}$, then the bound on $\left(M_{s, n, N}^{\tilde{R}}(t)-m(t)\right) / \sigma(t)$ is 0 , which is attained in the limit by continuous distribution function converging to the cdf of the form

$$
F(x)= \begin{cases}F_{0}(x), & \text { if } x<t \\ p, & \text { if } t \leq x<t+m(t) \\ 1, & \text { if } x \geq t+m(t)\end{cases}
$$

Now we use the integrand maximization-based approach to derive another bound (Papadatos 1997). This bound depends only $m(t)$ and then the previous assumption of finiteness of the second moment can be removed here.

The MRL of PCOSs can be rewritten as

$$
\begin{aligned}
M_{s, n, N}^{\tilde{R}}(t) & =\mathbb{E}\left(X_{s: n: N}^{\tilde{R}}-t \mid X_{s: n: N}^{\tilde{R}}>t\right) \\
& =\int_{t}^{\infty} \frac{1-F_{s: n: N}^{\tilde{R}}(F(x))}{1-F_{s: n: N}^{\tilde{R}}(p)} d x \\
& =\frac{1-p}{1-F_{s: n: N}^{\tilde{R}}(p)} \int_{p<F(x)<1} \frac{1-F_{s: n: N}^{\tilde{R}}(F(x))}{1-F(x)} \frac{1-F(x)}{1-p} d x \\
& \leq \frac{1-p}{1-F_{s: n: N}^{\tilde{R}}(p)} \sup _{p<u<1}\left(\frac{1-F_{s: n: N}^{\tilde{R}}(u)}{1-u}\right) m(t) .
\end{aligned}
$$

The function to be maximized in (17) represents the slopes of the straight lines joining the points of the graph of $F_{s: n: N}^{\tilde{R}}$ at $u$ and 1 . It increases on $\left(0, u_{s, n, N}\right)$ and then 
decreases on $\left(u_{s, n, N}, 1\right)$ with a maximal value at $u=u_{s, n, N}$. In the spirit of these lines, we have

$$
\sup _{p<u<1}\left(\frac{1-F_{s: n: N}^{\tilde{R}}(u)}{1-u}\right)= \begin{cases}(1-p)^{N-1}, & \text { if } s=1, \\ \prod_{i=1}^{n-1} \frac{\gamma_{i}}{\gamma_{i}-1}, & \text { if }\left(s=n, \gamma_{n}=1\right) \\ f_{s: n: N}^{R}\left(u_{s, n, N}\right), & \text { if }(1<s<n) \text { or }\left(s=n \geq 2, \gamma_{n}>1\right) \\ & \text { with } p<u_{s, n, N} \\ \frac{1-F_{s: n: N}^{\tilde{R}}(p)}{1-p}, & \text { if }(1<s<n) \text { or }\left(s=n \geq 2, \gamma_{n}>1\right) \\ & \text { with } p \geq u_{s, n, N .}\end{cases}
$$

Plugging the extreme values into (17), we readily establish the new bound. For either $(1<s<n)$ or $\left(s=n \geq 2, \gamma_{n}>1\right)$ with $p<u_{s, n, N}$, the inequality becomes equality if $F(x)=u_{s, n, N}$ or $F(x)=1$ for $x>t$. That is, we have two jumps $\left(u_{s, n, N}-p\right)$ and $\left(1-u_{s, n, N}\right)$ at $t$ and some point $\eta>t$, respectively. Using the moment condition, the value of $\eta$ is found to be

$$
\eta=t+\frac{1-p}{1-u_{s, n, N}} m(t) .
$$

If either $(1<s<n)$ with $p \geq u_{s, n, N}$ or $\left(s=n \geq 2, \gamma_{n}>1\right)$ with $p \geq u_{s, n, N}$ or $s=1$, the bounds here are the same as the ones in Theorem 3. So the attainability conditions in Theorem 3 are applied here. For $\left(s=n, \gamma_{n}=1\right)$, we should take the distribution that have values $F(x)$ close to 1 if $x>t$ and $F(x)<1$. We may assume $F(x)=1-\vartheta$ for $\{x: p<F(x)<1\}$. The moment condition implies that the distribution function has the jump from $1-\vartheta$ to 1 at $\eta=t+\frac{1-p}{\vartheta} m(t)$. The bounds obtained as well as the marginal distributions attaining these bounds are described in Theorem 4 below.

Theorem 4. For any continuous parent distribution $F$ having a finite mean $\mu$, we have for every point $t>0$ from the support of $F$,

$$
\begin{aligned}
& \frac{M_{s, n, N}^{\tilde{R}}(t)}{m(t)} \leq B_{2}^{\tilde{R}}(s, n, N ; p) \\
&= \begin{cases}\frac{(1-p)}{1-F_{s: n: N}^{\tilde{R}}(p)} \prod_{i=1}^{n-1} \frac{\gamma_{i}}{\gamma_{i}-1}, & \text { if }\left(s=n, \gamma_{n}=1\right), \\
\frac{(1-p) f_{s: n: N}^{\tilde{R}}\left(u_{s, n, N}\right)}{1-F_{s: n: N}^{\tilde{R}}(p)}, & \text { if }(1<s<n) \text { or }\left(s=n \geq 2, \gamma_{n}>1\right) \\
1, \quad & \text { with } p<u_{s, n, N}, \\
& \text { if } \begin{array}{l}
s=1 \text { or }(1<s<n) \text { with } p \geq u_{s, n, N} \\
\text { or }\left(s=n \geq 2, \gamma_{n}>1\right) \text { with } p \geq u_{s, n, N} .
\end{array}\end{cases}
\end{aligned}
$$

The bounds are attained in the limit by continuous distribution functions converging to the following distribution functions. If $1<s<n$ and $p<u_{s, n, N}$, the limiting distribution is of the form

$$
F(x)= \begin{cases}F_{0}(x), & \text { if } x<t \\ u_{s, n, N}, & \text { if } t \leq x<t+\frac{1-p}{1-u_{s, n, N}} m(t) \\ 1, & \text { if } x \geq t+\frac{1-p}{1-u_{s, n, N}} m(t)\end{cases}
$$


If $\left(s=n, \gamma_{n}=1\right)$, then the bound is attained in limit by continuous approximations of the family of distribution functions

$$
F(x)= \begin{cases}F_{0}(x), & \text { if } x<t, \\ 1-\vartheta, & \text { if } t \leq x<t+\frac{1-p}{\vartheta} m(t), \\ 1, & \text { if } x \geq t+\frac{1-p}{\vartheta} m(t),\end{cases}
$$

as $\vartheta$ tends to 0. Finally, if either $s=1$ or $(1<s<n)$ with $p \geq u_{s, n, N}$ or $\left(s=n \geq 2, \gamma_{n}>1\right)$ with $p \geq u_{s, n, N}$, then the limiting distribution is (16).

\section{Numerical experiments and discussion}

In this section, we have conducted a numerical study to evaluate the resulting bounds on the MRL of the PCOSs $\left(M_{s, n, N}^{\widetilde{R}}(t)-m(t)\right) / \sigma(t)$. The bounds are evaluated for $s=3,5, n=5$ and $N=10,20$ with different censoring schemes and $p=0,0.1,0.2,0.3$. Clearly, large values of $p$ need not to be considered here since if $p$ is getting large $\left(p \geq u_{s, n, N}\right)$, the bounds are reduced to the trivial minimal values.

The numbers $\varepsilon_{s, n, N}$ 's and $u_{s, n, N}$ 's can be obtained by solving (4) and (5). The solutions of (4) and (5) are determined numerically by means of the Newton-Raphson method. The solutions are plugged in (10) and (18) and obtain the final results. For the extreme cases, the bounds do not depend on $u_{s, n, N}$ 's and their evaluations can be performed directly. The results are presented in Tables 1 and 2 . In Table 1, we compare the bounds $B_{1}^{\tilde{R}}(s, n, N ; p)$ presented in Theorems 1,2 and 3 with the rough ones $B_{0}^{\tilde{R}}(s, n, N ; p)$. The bounds are identical for $\left(s=n, \gamma_{n}=1\right)$ [See, for example, the bounds for $(N=10, s=5, \widetilde{R}=$ $(5,0,0,0,0))$ and $(N=20, s=5, \widetilde{R}=(15,0,0,0,0))]$. The bounds combined with the Moriguti's method $B_{1}^{\tilde{R}}(n, n, N ; p)$ compare very well with the classical bounds derived by direct application of the Cauchy-Schwarz inequality, $B_{0}^{\tilde{R}}(n, n, N ; p)$. For fixed censoring

Table 1 Values of $B_{0}^{\tilde{R}}(s, 5, N ; p)$ and $B_{1}^{\tilde{R}}(s, 5, N ; p)$ for different censoring schemes and $p$

\begin{tabular}{|c|c|c|c|c|c|c|c|c|c|c|}
\hline \multirow[b]{2}{*}{$N$} & \multirow[b]{2}{*}{$s$} & \multirow[b]{2}{*}{$\tilde{R}$} & \multicolumn{2}{|l|}{$p=0$} & \multicolumn{2}{|l|}{$p=0.1$} & \multicolumn{2}{|l|}{$p=0.2$} & \multicolumn{2}{|l|}{$p=0.3$} \\
\hline & & & $\overline{B_{0}^{\tilde{R}}(.)}$ & $B_{1}^{\tilde{R}}()$. & $\overline{B_{0}^{\tilde{R}}(.)}$ & $B_{1}^{\tilde{R}}()$. & $\overline{B_{0}^{\tilde{R}}(.)}$ & $B_{1}^{\tilde{R}}()$. & $\overline{B_{0}^{\tilde{R}}(.)}$ & $B_{1}^{\tilde{R}}()$. \\
\hline \multirow[t]{4}{*}{10} & 3 & $(2,0,0,0,3)$ & 0.9689 & 0.2148 & 0.9389 & 0.0000 & 1.0455 & 0.0000 & 1.1663 & 0.0000 \\
\hline & & $(5,0,0,0,0)$ & 0.6591 & 0.3130 & 0.5722 & 0.1235 & 0.5711 & 0.0009 & 0.6218 & 0.0000 \\
\hline & & $(1,1,1,1,1)$ & 0.9976 & 0.2069 & 0.9750 & 0.0000 & 1.0924 & 0.0000 & 1.2170 & 0.0000 \\
\hline & & $(0,0,3,0,2)$ & 1.1087 & 0.1848 & 1.1130 & 0.0000 & 1.2702 & 0.0000 & 1.4162 & 0.0000 \\
\hline \multirow[t]{4}{*}{10} & 5 & $(2,0,0,0,3)$ & 0.8555 & 0.5342 & 0.7486 & 0.3976 & 0.6468 & 0.2287 & 0.6017 & 0.0628 \\
\hline & & $(5,0,0,0,0)$ & 1.2341 & 1.2341 & 1.1273 & 1.1273 & 1.0103 & 1.0103 & 0.8830 & 0.8830 \\
\hline & & $(1,1,1,1,1)$ & 0.7593 & 0.6325 & 0.6477 & 0.5105 & 0.5256 & 0.3651 & 0.4203 & 0.2102 \\
\hline & & $(0,0,3,0,2)$ & 0.7957 & 0.5426 & 0.6865 & 0.4077 & 0.5800 & 0.2429 & 0.5253 & 0.0808 \\
\hline \multirow[t]{4}{*}{20} & 3 & $(5,4,3,2,1)$ & 1.3773 & 0.1325 & 1.4928 & 0.0000 & 1.7098 & 0.0000 & 1.8312 & 0.0000 \\
\hline & & $(15,0,0,0,0)$ & 0.6463 & 0.2671 & 0.5758 & 0.0629 & 0.6014 & 0.0000 & 0.6571 & 0.0000 \\
\hline & & $(3,3,3,3,3)$ & 1.5385 & 0.1182 & 1.7214 & 0.0000 & 1.9888 & 0.0000 & 2.1359 & 0.0000 \\
\hline & & $(5,0,5,0,5)$ & 1.5285 & 0.1199 & 1.7054 & 0.0000 & 1.9769 & 0.0000 & 2.1345 & 0.0000 \\
\hline \multirow[t]{4}{*}{20} & 5 & $(5,4,3,2,1)$ & 0.6666 & 0.4871 & 0.5504 & 0.3407 & 0.4493 & 0.1700 & 0.4206 & 0.0312 \\
\hline & & $(15,0,0,0,0)$ & 1.1842 & 1.1842 & 1.0780 & 1.0780 & 0.9618 & 0.9618 & 0.8363 & 0.8363 \\
\hline & & $(3,3,3,3,3)$ & 0.9126 & 0.3631 & 0.8155 & 0.1675 & 0.8067 & 0.0000 & 0.8982 & 0.0000 \\
\hline & & $(5,0,5,0,5)$ & 1.0145 & 0.3474 & 0.9206 & 0.1413 & 0.9340 & 0.0000 & 1.05954 & 0.0000 \\
\hline
\end{tabular}


Table 2 Values of $B_{2}^{\tilde{R}}(s, 5, N ; p)$ for different censoring schemes and $p$

\begin{tabular}{|c|c|c|c|c|c|c|}
\hline \multirow[b]{2}{*}{$N$} & \multirow[b]{2}{*}{$s$} & \multicolumn{5}{|c|}{$B_{2}^{\tilde{R}}(s, 5, N ; p)$} \\
\hline & & $\tilde{R}$ & $p=0$ & $p=0.1$ & $p=0.2$ & $p=0.3$ \\
\hline \multirow[t]{4}{*}{10} & 3 & $(2,0,0,0,3)$ & 1.0611 & 1.0000 & 1.0000 & 1.0000 \\
\hline & & $(5,0,0,0,0)$ & 1.1355 & 1.0377 & 1.0000 & 1.0000 \\
\hline & & $(1,1,1,1,1)$ & 1.0566 & 1.0000 & 1.0000 & 1.0000 \\
\hline & & $(0,0,3,0,2)$ & 1.0448 & 1.0000 & 1.0000 & 1.0000 \\
\hline \multirow[t]{4}{*}{10} & 5 & $(2,0,0,0,3)$ & 1.3572 & 1.2222 & 1.0992 & 1.0169 \\
\hline & & $(5,0,0,0,0)$ & 4.4444 & 4.0001 & 3.5575 & 3.1231 \\
\hline & & $(1,1,1,1,1)$ & 1.5260 & 1.3737 & 1.2282 & 1.1064 \\
\hline & & $(0,0,3,0,2)$ & 1.3733 & 1.2366 & 1.1117 & 1.0249 \\
\hline \multirow[t]{4}{*}{20} & 3 & $(5,4,3,2,1)$ & 1.0228 & 1.0000 & 1.0000 & 1.0000 \\
\hline & & $(15,0,0,0,0)$ & 1.0994 & 1.0143 & 1.0000 & 1.0000 \\
\hline & & $(3,3,3,3,3)$ & 1.0180 & 1.0000 & 1.0000 & 1.0000 \\
\hline & & $(5,0,5,0,5)$ & 1.0185 & 1.0000 & 1.0000 & 1.0000 \\
\hline \multirow[t]{4}{*}{20} & 5 & $(5,4,3,2,1)$ & 1.3062 & 1.1769 & 1.0676 & 1.0071 \\
\hline & & $(15,0,0,0,0)$ & 4.2105 & 3.7896 & 3.3712 & 2.9631 \\
\hline & & $(3,3,3,3,3)$ & 1.1607 & 1.0497 & 1.0000 & 1.0000 \\
\hline & & $(5,0,5,0,5)$ & 1.1458 & 1.0374 & 1.0000 & 1.0000 \\
\hline
\end{tabular}

scheme, as $p$ increases, the bounds decrease in $p$, and become zero if $p \geq u_{s, n, n}$. It is not surprising to observe that while we can't see a regular behavior for $B_{0}^{\tilde{R}}(s, n, N ; p)$, the Moriguti's bound increases when $s$ increases.

Table 2 presents numerical values of bounds $B_{2}^{\tilde{R}}(n, n, N ; p)$ of Theorem 4 . The bounds of PCOSs here are represented in the scale units $\mathrm{m}(\mathrm{t})$. The values of the bounds take the maximized values when $p=0$ and then decrease to the minimum value when $p$ gets large. It is observed that the large bound occurs when $\left(s=n, \gamma_{n}=1\right)$.

It is of interest to point out that the two estimates of the MRL of PCOSs $M_{s, n, N}^{\widetilde{R}}(t)$ presented in Tables 1 and 2 are quite different. For comparison purposes, we have to rewrite both bounds as follows:

$$
\begin{aligned}
& M_{s, n, N}^{\widetilde{R}}(t) \leq B_{11}^{\tilde{R}}(s, n, N ; p)=m(t)+B_{1}^{\tilde{R}}(s, n, N ; p) \sigma(t), \\
& M_{s, n, N}^{\widetilde{R}}(t) \leq B_{22}^{\tilde{R}}(s, n, N ; p)=B_{2}^{\tilde{R}}(s, n, N ; p) m(t) .
\end{aligned}
$$

The former bound competes the latter one iff

$$
m(t) \geq \frac{B_{1}^{\tilde{R}}(s, n, N ; p)}{B_{2}^{\tilde{R}}(s, n, N ; p)-1} \sigma(t) .
$$

This means that the mean-variance bound $B_{11}^{\tilde{R}}(s, n, N ; p)$ compares well with respect to $B_{22}^{\tilde{R}}(s, n, N ; p)$ when the residual mean $m(t)$ is large with respect to the residual standard deviation. Let illustrate that via a specific example. We calculate bounds $B_{11}^{\tilde{R}}(s, n, N ; p)$ and $B_{22}^{\tilde{R}}(s, n, N ; p)$ in the cases $(m(t)=0.5, \sigma(t)=1)$ and $(m(t)=3, \sigma(t)=0.25)$. In the first case, we obtain $B_{11}^{\tilde{R}}(3,5,10 ; 0.1)=0.6235>B_{22}^{\tilde{R}}(3,5,10 ; 0.1)=0.5189$, whereas for the latter case, we have $B_{11}^{\tilde{R}}(3,5,10 ; 0.1)=3.0309<B_{22}^{\tilde{R}}(3,5,10 ; 0.1)=3.1133$. It assures the superiority of Moriguti projection method over maximization-based method 
[Papadatos (1997)] when $m(t)$ is large with respect to $\sigma(t)$. The result is reversed when $m(t)$ tends to be small when compared to $\sigma(t)$.

\section{Concluding remarks}

In this paper, we have developed the maximized evaluations of the MRL function of PCOSs of order statistics, measured in location and scale units of the residual life random variable $X_{t}=(X-t \mid X>t)$ and expressed in terms of percentile points $t=F^{-1}(p), 0<p<1$. The projection based approach as well as the maximum functional based approach method are used to obtain two different bounds. It is shown that the mean-variance bound derived based on the projection method competes well when compared with the ordinary bounds obtained via Cauchy-Schwarz inequality. For comparing the two so obtained bounds, we recommend choosing the mean-variance bound if we can assume that the mean residual life is large with respect to the residual variance.

Similar results can be done for the second concept of the MRL of PCOSs, $M_{s, n}^{* \tilde{R}}(t)=$ $\mathbb{E}\left(X_{s: n: N}^{\tilde{R}}-t \mid X_{r: n: N}^{\tilde{R}} \leq t<X_{r+1: n: N}^{\tilde{R}}\right)$. Specifically, the bounds for $M_{s, n}^{*^{\tilde{R}}}(t)$ are identical to the optimal bounds for the unconditional expectation $E\left(X_{s-r: n-r: \gamma_{r+1}}\right)$, where $\tilde{S}=$ $\left(R_{r+1}, \ldots, R_{n}\right)$ (see, Lemma 2.1, Hashemi et al. (2010)). Accordingly, the optimal bounds for $M_{s, n}^{* \tilde{R}}(t)$ will be free of the parameter $p(0<p<1)$. Therefore, the results for the alternative concept of MRL of PCOSs $M_{s, n}^{* \tilde{R}}(t)$ are similar to those of progressive type II censored order statistics established in Raqab (2003). While the bounds do not depend on $p$, the probability distributions characterized via these bounds are described in terms of $p, m(t)$, and $\sigma(t)$.

\section{Acknowledgement}

The author would like to thank the referees for their suggestions and comments on the first draft of this manuscript.

Received: 19 June 2014 Accepted: 16 September 2014

V.

\section{References}

Abouammoh, A, El-Neweihi, E: Closure of the NBUE and DMRL classes under formation of parallel systems. Statist. Probab. Lett. 4, 223-225 (1986)

Arnold, BC: $p$-Norm bounds on the expectation of the maximum of a possibly dependent sample. J. Multivariate Anal. 17, 316-332 (1985)

Asadi, M, Bayramoglu, I: A note on the mean residual life function of a parallel system. Commun. Stat. Theory Methods 34(2), 475-485 (2005)

Asadi, M, Bayramoglu, I: The mean residual life function of a k-out-of- $n$ structure at the system level. IEEE Trans. Reliability 55(2), 314-318 (2006)

Bairamov, I, Ahsanullah, M, Akhundov, I: A Residual life function of a system having parallel or series structures. J. Stat. Theory Appl. 1(2), 119-132 (2002)

Balakrishnan, N, Aggarwala, R: Progressive Censoring. Birkhäuser, Boston (2000)

Balakrishnan, N, Cramer, E, Kamps, U: Bounds for means and variances of progressive type II censored order statistics. Statist. Probab. Lett. 54, 301-315 (2001)

Guess, F, Proschan, F: Mean residual life: theory and applications. In: Krishnaiha, PR, Rao, CR (eds.) Handbook of Statistics, vol. 7, pp. 215-224. Elsevier, Netherlands, (1988)

Hashemi, M, Tavangar, M, Asadi, M: Some properties of the residual lifetime of progressively Type-Il right censored order statistics. Statist. Probab. Lett. 80, 848-859 (2010)

Kamps, U, Cramer, E: On distributions of generalized order statistics. Statistics 35, 269-280 (2001)

Kotz, S, Shanbhag, DN: Some new approaches in probability distributions. Adv. Appl. Probability 12, 903-921 (1980)

$\mathrm{Li}, \mathrm{X}$, Chen, J: Aging properties of the residual life of $k$-out-of- $n$ systems with independent but non-identical components. Appl. Stochastic Models Business Ind. 20, 143-153 (2004)

Moriguti, S: A Modification of Schwarz's inequality with applications to distributions. Ann. Math. Statist. 24, 107-113 (1953)

Papadatos, N: Exact bounds for the expectations of order statistics from non-negative populations. Ann. Inst. Stat. Math. 49(4), 727-736 (1997)

Poursaeed, MH, Nematollahi, AR: On the mean past and the mean residual life under double monitoring. Commun. Stat. Theory Meth. 37, 1119-1133 (2008) 
Raqab, MZ: P-Norm bounds for moments of progressive type II censored order statistics. Statist. Probab. Lett. 64, 393-402 (2003)

Raqab, MZ, Rychlik, T: Bounds for the mean residual life function of a k-out-of- $n$ system. Metrika 74(3), 361-380 (2011)

Rychlik, T: Sharp bounds on L-estimates and their expectations for dependent samples. Commun. Statist. Theory Meth. 22, 1053-1068 (1993)

Sen, PK: Progressive Censoring Schemes. In: Kotz, S, Johnson, NL (eds.) Encyclopedia of Statistical Sciences, vol. 7, pp. 296-299. Wiley, New York, (1986)

doi:10.1186/s40488-014-0021-5

Cite this article as: Raqab: Bounds on the mean residual lifetime of progressive type II right censored order statistics. Journal of Statistical Distributions and Applications 2014 1:21.

\section{Submit your manuscript to a SpringerOpen ${ }^{\odot}$} journal and benefit from:

- Convenient online submission

- Rigorous peer review

- Immediate publication on acceptance

- Open access: articles freely available online

- High visibility within the field

- Retaining the copyright to your article

Submit your next manuscript at $\boldsymbol{\triangleright}$ springeropen.com 